\title{
1. The need for disruptive innovation in public services
}

\section{WHY STUDY PUBLIC SERVICES?}

Public services are an important part of all societies. Government spending constitutes a significant portion of gross domestic product (GDP) across the developed world. The Organisation for Economic Co-operation and Development (OECD) estimated the range of government share of GDP in 2009 at 18 to 58 percent. Individual countries show significant variance. As an example, the United States and Japan represent countries with a relatively small government share of the economy (approximately 37-42 percent) whereas the United Kingdom and Scandinavian countries range from 52 to 58 percent (OECD, 2011).

This variation comes into even greater relief when one compares the size and distribution of government expenditures by category. There is significant variation in spending and mix for health and social protection services. Compare Norway's commitment of more than 23 percent of budget to social and health issues, to the United States' 18 percent of budget which includes a much higher proportion allocated to healthcare. Figure 1.1 graphically depicts four countries' approaches to health and social service spending.

This data reflects not only different economic structures, but also different policy views on the roles of government, public services and the private sector. The 2005 OECD Ministerial Meeting on 'Strengthening Trust in Government: What Role for Government in the 21st Century?' concluded that 'a responsible government ... . works for the collective interest, and looks at the medium to long term to ensure that future generations are not short-changed' (OECD, 2011).

The balance that the OECD describes - meeting short term-service needs while preparing for medium- and long-term challenges - is not unlike mainstream strategic thought in the private sector. What 


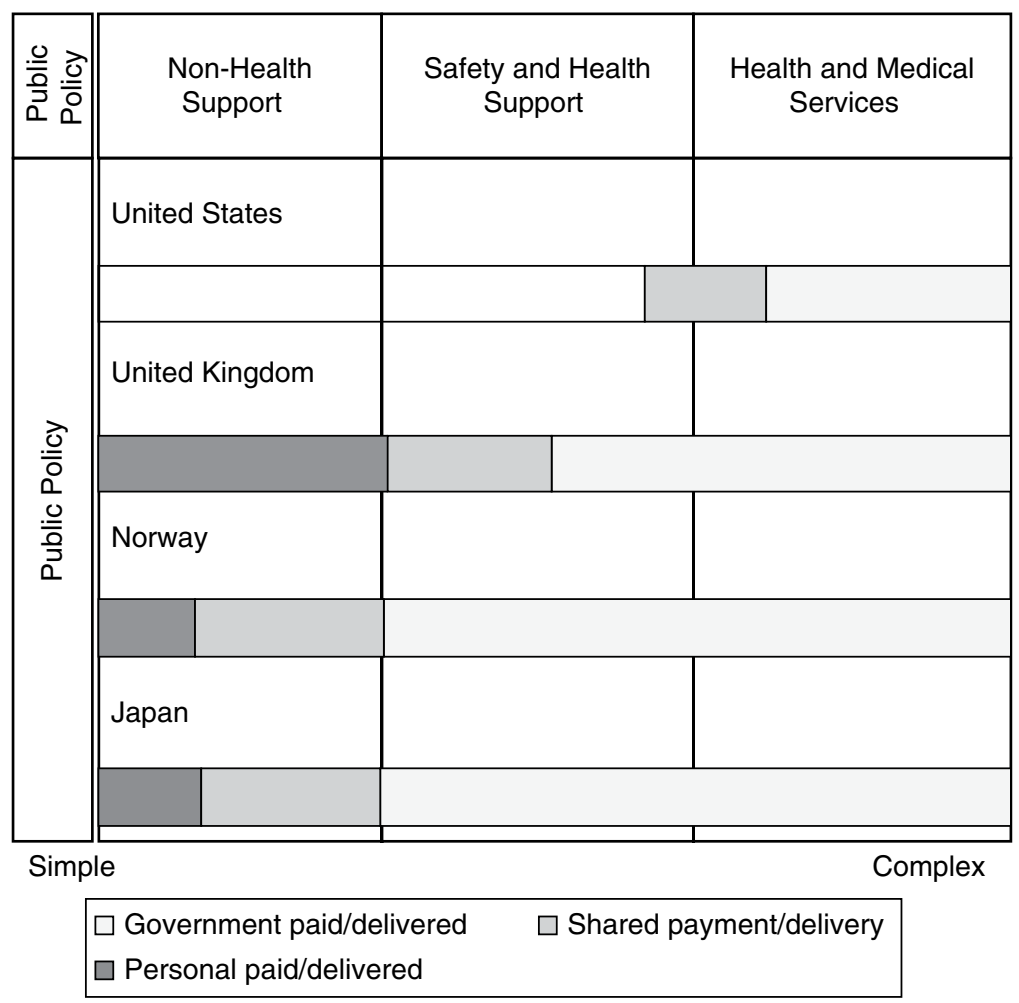

Figure 1.1 Public policy approaches to health and social services

is significantly different, and at the heart of public services, is the role of the citizen. Each citizen in a developed democratic society has three broad roles in public services: as purchaser, through direct payment for services (for oneself or others), or indirect payment through taxes; as consumer of direct services; and as a participant in a political process (through voting or other active involvement) that determines public policy. This interaction is illustrated in Figure 1.2.

Public policy creates the basis for service delivery, most notably through the allocation of resources and the creation of a regulatory infrastructure that can govern service operations. The results of that service delivery, when combined with an assessment of the current and expected future environment, are key inputs to the evolution of 


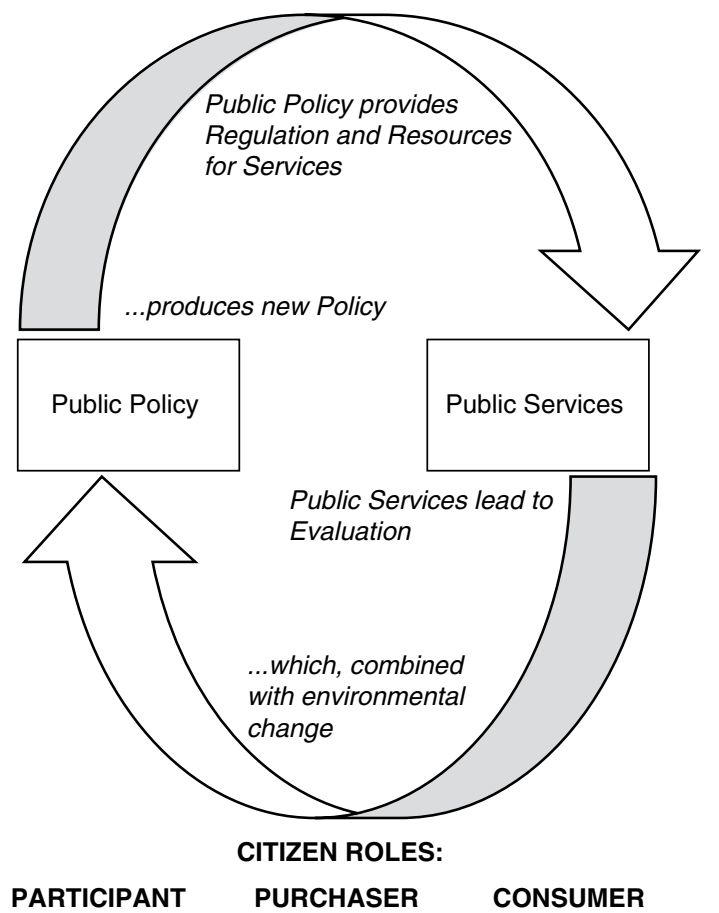

Figure 1.2 Citizen involvement in public services

public policy, creating a reinforcing loop over time. At any point in this cycle, we as individuals can be playing a combination of roles; collectively, we as a pluralistic society influence both public policy and service delivery.

One of the central questions raised by this model is how we accomplish the 'balancing act' of public policy and public service. Specifically, are decisions consistent between the political arena and the marketplace? The marketplace tends to be more concerned with short-term demands, while the political arena is, but will never fully be, concerned with longer-term public policy. If they were consistent, then it would appear reasonable to consider a single theory of innovation. However, if there is diversity modeling in decision-making that reflects both political and economic values, then we would do well to entertain a broader theory of innovation and allow for the idea that what one group or country considers highly innovative and 
desirable may be anathema to another. Our primary hypothesis is that the latter is the state of affairs and a critical 'litmus test', and so we have undertaken to build such a theory and an evidence base to explore it.

\section{WHY STUDY INDEPENDENT LIVING?}

While we believe that public services present a compelling set of questions in the abstract, we have chosen a single area as a primary filter and grounding for the discussion - in-home services to support independent living for seniors and the disabled. We believe that independent living is an excellent platform for understanding public service innovation for four reasons.

\section{There is a Compelling Need for Services}

The developed world is ageing at a rapid rate, which is generating exponential increases in demand for associated services: health care, mobility, transportation, safety, and social interaction to name but a few. Our gains in longevity are being offset by the accelerated growth in incidence and prevalence of chronic diseases - heart disease, stroke, cancer, pulmonary disease, depression, and the like. The disabling and long-term symptoms of chronic disease contribute to extended pain and suffering, and reduced quality of life.

There is good reason to believe that our capacity to deliver within the current model of health care - especially reliance on licensed professional caregivers operating from dedicated, high-cost medical facilities - will be severely limited in the future. The forecasts are even more dire for non-professional workers. This combination of ageing, disease incidence, and caregiver shortages creates a compelling need for service.

\section{Independent Living is a Focus of Potentially Disruptive, Technology-enabled Services}

We face a future in which demand for care services will explode precisely when our resources are least able to meet that demand. One implication of this situation is that the nature and scale 
of the potential solutions must be larger and more disruptive. Such strategies as treating in homes and the community, involving non-professional caregivers, and using technology are very consistent with the evolution of in-home care models.

Many countries are looking to technological innovation to support more accessible and affordable independent living. The technologies of focus, for which language is still evolving (Martin, 2010), fall under three broad categories: telecare, telehealth, and 'smart home' technology.

\section{The Social and Economic Consequences of Independent Living are Driving Business Models and Public Policy}

The cost and power implications of economic support for independent living are generating substantial and increasingly conflicted debate. There is an active discussion in many countries surrounding the philosophical and practical underpinnings of 'entitlement programs'. Among the foci of those discussions are the proposal of fundamental shifts in determining and administering benefits. In chronic disease care, new business models are emerging in response to public and private policies designed to improve outcomes and reduce spending. Some of these models establish conditions that foster the adoption of enabling technologies. Collectively, advocates of these innovations propose that they offer the potential for disruptive support of independent living by ensuring that seniors can spend 'one more year at home' - then another, and another.

\section{Adoption is Slow, and Regulation is Problematic}

Despite the theoretical advantages and generally positive evidence attached to such service models, there is little compelling scientific evidence of improved outcomes, and adoption is slow. There are a number of reasons cited for this pattern: misaligned payment systems, barriers to use of technology by the elderly, privacy concerns, and the like. In addition most providers have little experience with remote clinical technologies. They are poorly prepared to evaluate the technologies or to make decisions about their acquisition or deployment (Coye et al., 2009). 


\section{CURRENT THEORY HAS GAPS IN UNDERSTANDING}

We need powerful theory and tools to address critical societal issues such as public service design and delivery. Our survey of theories and tools, undertaken long before the genesis of this book, has highlighted important gaps in understanding. (The relevant theories and analysis are presented in detail in Part III of this book.)

Innovation theory and tools, such as those of Doblin (Keeley et al., 2013) and Osterwalder (Osterwalder and Pigneur, 2010), have been largely rooted in the private sector. As such, they focus intensely on internal strategy, operations, and design of the customer experience. They are largely silent, however, on external factors such as public policy and culture.

Cultural analysis has a well-established set of tools for both internal and comparative purposes; however, there is little or no integration of practical strategy and operational elements that are so often at the core of successful service launch and delivery. Finally, there is very little scholarship on the subject of integrating tools and viewpoints across disciplines.

Our own theoretical analysis is grounded in a different model of innovation: the Service Innovation Triangle (SIT) model (Furseth and Cuthbertson, 2016). The original SIT model is a broad framework whose greatest strength is in addressing the complexity of service innovation in a clear way, and offering a tool to explore a rich variety of potential explanations for the success or failure of a particular service or provider.

It has also proven to be an excellent framework within which one can explore social and cultural issues. We have supplemented the original SIT model with social and cultural factors. We believe that such an integrated model offers great benefits for public agencies seeking to improve performance, private entities seeking entry into public sector services, and societies looking for the most effective ways of advancing their work. The result is a more comprehensive and powerful approach to understanding public sector innovation that broadens and deepens the dimensions that shape both the determination and implementation of crucial public programs. 


\section{CONCLUSION}

The importance of public services to society in general and the critical role of independent living in coping with the rapid ageing of developed and developing countries underscore the potential benefits of creating truly disruptive public services innovation. Current innovation theory, while useful for understanding basic dynamics, is not sufficiently nuanced, particularly in matters of culture, to comprehend the full range of economic, social, and political issues that bear on major public policy. This book is an attempt to develop and test a broader theory of public service innovation that can improve the level of public service innovation, and thus the performance of public services in this critical area. 